\title{
Pnictide-height dependent ferromagnetism in CuFeAs and CuFeSb
}

\author{
Guangtao Wang*, Xianbiao Shi, and Dongyang Wang \\ College of Physics and Electronic Engineering, Henan Normal University, \\ Xinxiang, Henan 45300\%, People's Republic of China
}

(Dated: May 30, 2016)

\begin{abstract}
Electronic structures and magnetism properties of $\mathrm{CuFeAs}$ and $\mathrm{CuFeSb}$ are investigated by using first-principles calculations. We found that $\mathrm{CuFeAs}$ and $\mathrm{CuFeSb}$ share similar electronic structures and magnetic properties. Unlike the antiferromagnetic isostructure LiFeAs, the ground state of both compounds is ferromagnetic state driven by the Stoner ferromagnetic instability. Their ground state is very sensitive to the height of anion (As or Sb), translating from the ferromagnetic state to the stripe antiferromagnetic ordering when the anion height is smaller than a critical value. Such magnetic phase transition can be understood by the $J_{1}-J_{2}$ Heisenberg model. Reducing the anion height will decrease the nearest-neighbor interaction $J_{1}$ but increase the next-nearest-neighbor interaction $J_{2}$. The competing between the anion height dependent antiferromagnetic superexchange mediated by $\mathrm{As}(\mathrm{Sb})$ and the ferromagnetic direct exchange between Fe results the variations of magnetic structure with anion height.
\end{abstract}

Keywords: Superconductors, Electronic properties, Magnetic properties

\section{INTRODUCTION}

The emergence of superconductivity with high transition temperature (Tc) in ferropnictide ${ }^{1-6}$ and ferrochalcogenide ${ }^{7-10}$ is helpful for understanding the mechanism of high-Tc Superconductors. The similar phase diagram to copper oxides, where superconductivity develops when magnetic order is destroyed by doping or high pressure, suggests a strong interplay between superconductivity and magnetism ${ }^{11,12}$. It is generally accepted that the superconductivity in iron based superconductors is dominated by magnetism-mediated rather than phonon-mediated pairing. Therefore, it is important to study the mechanism of magnetism in Fe-based superconductors. To understand the magnetism puzzle, the scenarios based on both itinerant weak-coupling picture and the localized strong-coupling picture have been constructed. In the first picture, the magnetism can be understood by the Fermi surface (FS) nesting ${ }^{11,13-15}$. However, this picture failed to explain the double-stripe antiferromagnetic $(\mathrm{AF})^{16}$ ground state of FeTe, whose FS topology is similar to iron pnictides ${ }^{17}$. In the localized strong-coupling picture, the magnetic order is explained by the frustrated super-exchange interactions and the Heisenberg model for local spin moments ${ }^{18-22}$. Nevertheless, this model can not explain the metallic resistivity and is incompatible with the large anisotropy of the nearest-neighbor exchange constant ${ }^{23}$. Apart from the above opposite viewpoints, a new theory with coupling the local moments and itinerant electrons has also been considered by Johannes ${ }^{24}$ and $\mathrm{Yin}^{25}$.

\footnotetext{
* Corresponding author.

E-mail addresses: wangtao@henannu.edu.cn
}

By first-principles calculations, $\mathrm{Xu}$ et $a l^{26}$ presented a doping-dependent phase diagram of the LaOMAs $(\mathrm{M}=$ $\mathrm{V}-\mathrm{Cu}$ ) family, and found that the Fe-based compound was at the borderline between the AF and ferromagnetic (FM) phase regions. Yin et $a l^{25}$ unified the FM and $\mathrm{AF}$ interactions in the microscopic model, where they assume that the superexchange AF interaction competes with the orbital-degenerated double-exchange FM interaction. With the increment of the anion height from the iron plane $\left(Z_{\text {anion }}\right)$, the magnetic ground state evolve from the stripe AF order to the double-stripe AF order, and finally to the FM order. Moon's 27 calculations on $\mathrm{FeTe}_{1-x} \mathrm{Se}_{x}$ demonstrated that its ground state switched between stripe AF and double-stripe AF order with varying $Z_{\text {anion }}$, they also found the tendency toward FM state if the value of $Z_{\text {anion }}$ was large enough.

Recently, two new layered ferropnictide compounds with formula as $\mathrm{CuFeAs}{ }^{28,29}$ and $\mathrm{CuFeSb}^{30}$ were synthesized, with their structures being similar to the layered tetragonal 111-type iron-based superconductors LiFeAs ${ }^{3}$ and $\mathrm{NaFeAs}^{4}$. The anions height is $\mathrm{Z}_{A s}=1.74$ (1.81) $\AA$ in $\mathrm{CuFeAs}^{28,29}$ and $\mathrm{Z}_{S b}=1.84 \AA$ in $\mathrm{CuFeSb}^{30}$, which is significantly larger than $\mathrm{Z}_{\text {anion }}=1.31,1.35,1.51$, 1.47, and $1.73 \AA$ in $\mathrm{LaOFeAs}^{1}, \mathrm{BaFe}_{2} \mathrm{As}_{2}{ }^{2}, \mathrm{LiFeAs}^{3}$, $\mathrm{FeSe}^{7}$ and $\mathrm{FeTe}^{8}$, respectively. Using magnetization and neutron scattering measurements, Qian et $a l^{30}$ discover that the ground state of $\mathrm{CuFeSb}$ is metallic FM state. The magnetic properties of CuFeAs is under debate: Qian et $a l^{29}$ reported that its ground state was FM, while Thakur et $a l^{28}$ claimed the AF state. Until now, no first-principle calculations about $\mathrm{CuFeAs}$ and $\mathrm{CuFeSb}$ have been reported. In this paper we employed the first-principles technique to investigate the electronic structures and magnetism of these compounds. 

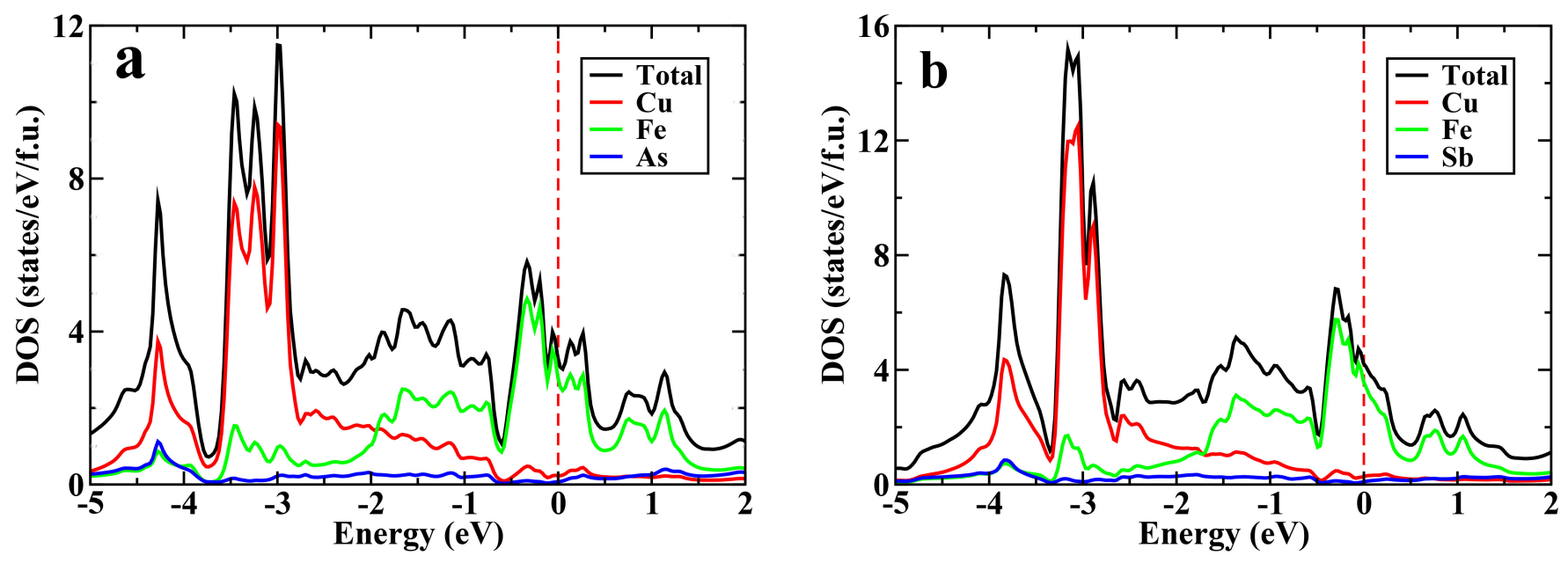

FIG. 1: (Color online) The DOS and projected DOS of (a) CuFeAs and (b) CuFeSb in the NM configure, with GGA formula.

\section{METHODOLOGY}

The calculations were performed by using STATE $^{31}$ code based on the density functional theory (DFT). The generalized gradient approximation (GGA) ${ }^{32}$ with the Perdew-Burke-Ernzerhof (PBE) ${ }^{33}$ formula for the exchange-correlation potentials was used in the calculation. All the lattice constants and atomic positions of both compounds were taken from experiments ${ }^{28,30}$. To determine the true ground state, we considered four different cases: nonmagnetic (NM), FM, and the two AF spin configurations. The first one is AF1 (checkerboard AF), where the nearest-neighbor Fe spins are antiparallel in the $a-b$ plane and ferromagnetic along the c-axis. The second one is AF2 (stripe AF), where the Fe spins along the square diagonal are aligned antiferromagnetically. The same unit cell was used for the NM, FM, and AF1 states, while we considered the unit cell as $\sqrt{2} a \times \sqrt{2} a \times c$ for the AF2 state. After carefully examining the convergence with respect to the cutoff energy and number of k-points, we adopted a cutoff energy of $30 \mathrm{Ry}$ and the Monkhorst-pack k-points generated with $20 \times 20 \times 16$ grids for the NM, FM and AF 1 states and $16 \times 16 \times 12$ grids for the AF2 state.

\section{RESULTS AND DISCUSSION}

Fig. 1(a) shows the density of states (DOS) and projected DOS of CuFeAs in the NM state. The $\mathrm{Cu}-3 \mathrm{~d}$ states mainly lie between -5.0 and $-2.0 \mathrm{eV}$, accompanying with the empty $4 \mathrm{~s}$ state above the Fermi level $\left(E_{F}\right)$. Therefore, the $\mathrm{Cu} 3 \mathrm{~d}$ orbits are fully occupied with $d^{10}$ shell at high binding energy, similar to the situation in $\mathrm{ThCr}_{2} \mathrm{Si}_{2}$ structural $\mathrm{ACu}_{2} \mathrm{As}_{2}(\mathrm{~A}=\mathrm{Ca}, \mathrm{Sr}, \mathrm{Ba})^{34}$. Just like the Fe-pnictide superconductors, the states around the $E_{F}$ (from -2.0 to $2.0 \mathrm{eV}$ ) are mostly the Fe-3d states.
At the $E_{F}$, the DOS is $\mathrm{N}\left(\mathrm{E}_{F}\right)=3.3 \mathrm{eV}^{-1}$ per formulae. From $\mathrm{N}\left(\mathrm{E}_{F}\right)$, the calculated bare susceptibility and specific heat coefficient are $\chi_{0}=1.07 \times 10^{-4} \mathrm{emu} / \mathrm{mol}$ and $\gamma_{0}=7.85 \mathrm{~mJ} /\left(\mathrm{K}^{2} \cdot \mathrm{mol}\right)$, respectively. Our calculated specific heat coefficient is only $\frac{1}{5}$ as the experimental value $\gamma_{0}=40 \mathrm{~mJ} /\left(\mathrm{K}^{2} \cdot \mathrm{mol}\right)^{29}$. Such high value may be due to nearness to a magnetic quantum critical point ${ }^{29}$. In Figs.1(a) and 1(b), the Fe-3d contribution to $\mathrm{N}\left(\mathrm{E}_{F}\right)$ is more than $3 \mathrm{eV}^{-1}$ per atom, which places the material near teh Stoner itinerant magnetism due to the Stoner criterion $^{35}: \mathrm{N}\left(\mathrm{E}_{F}\right) \mathrm{I}>1$ ( $\mathrm{I}$ is the Stoner parameter of Fe $\left.\mathrm{I} \sim 0.7-0.8^{36}\right)$.

Comparing the DOS of CuFeSb [Fig. 1(b)] with that of $\mathrm{CuFeAs}$, we find that the $\mathrm{p}$-d hybridization between Sb-5p states and Fe-3d states is weaker than the hybridization between Fe-3d and As-4p. This enhances the itinerant character of $\mathrm{CuFeSb}$. The larger $\mathrm{Z}_{S b}$ may be responsible for such $\mathrm{p}$-d hybridization reduction. Besides, it is interesting to note that the Fe bandwidth of $\mathrm{CuFeSb}$ is $0.3 \mathrm{eV}$ narrower than that of $\mathrm{CuFeAs}$, due to the longer $\mathrm{Fe}-\mathrm{Fe}$ distance in the Sb compound (2.782 A vs $2.648 \AA$ ). Such band narrowing may enhance the magnetic Fe moment in $\mathrm{CuFeSb}$.

The projected band structure of $\mathrm{CuFeAs}$ is presented in Fig.2, where the symbol size corresponds to the projected weight of Bloch states onto the Fe (a) $\mathrm{d}_{r^{2}-3 z^{2}}$, (b) $\mathrm{d}_{x^{2}-y^{2}}$, (c) $\mathrm{d}_{x y}$, and (d) $\mathrm{d}_{y z / z x}$ orbits. There are four bands (two hole-like bands around the $\Gamma(0,0,0)$ point and two electron-like bands around the $\mathrm{M}(\pi, \pi, 0))$ crossing the $\mathrm{E}_{F}$, which is in consistence with the metallic resistance. The band dispersion along symmetry lines near the $\mathrm{E}_{F}$ quite resembles to that of LiFeAs and $\mathrm{MgFeGe}^{37}$, where the former is a superconductor and the later is a ferromagnetic metal ${ }^{38}$. The bands around $\mathrm{E}_{F}$ (from -1.0 to $1.0 \mathrm{eV}$ ) are mostly derived from $\mathrm{d}_{x y}$ and $\mathrm{d}_{y z / z x}$ orbits [Figs. $2(\mathrm{c})$ and $2(\mathrm{~d})$ ], while the states 
TABLE I: Calculated total energies (with respect to the NM state), magnetic moments of Fe atoms, and DOS at $E_{F}\left[N\left(E_{F}\right)\right]$ of $\mathrm{CuFeAs}$ and $\mathrm{CuFeSb}$ in the NM, FM, checkerboard, and stripe AF states respectively. The energy of NM state is set to zero.

\begin{tabular}{l|ccc|ccc}
\hline \hline & \multicolumn{3}{|c|}{ CuFeAs } & \multicolumn{3}{c}{ CuFeSb } \\
\hline Order & Energy $(\mathrm{meV})$ & Moment $\left(\mu_{B}\right)$ & $\mathrm{N}\left(\mathrm{E}_{F}\right)\left(\mathrm{eV}^{-1}\right)$ & Energy $(\mathrm{meV})$ & Moment $\left(\mu_{B}\right)$ & $\mathrm{N}\left(\mathrm{E}_{F}\right)\left(\mathrm{eV}^{-1}\right)$ \\
NM & 0 & 0 & 3.3 & 0 & 0 & 3.97 \\
FM & -213.21 & 2.27 & 2.91 & -293.79 & 2.36 & 1.98 \\
AF1 & -84.09 & 1.86 & 4.18 & -156.92 & 2.07 & 3.57 \\
AF2 & -151.85 & 1.84 & 3.48 & -215.58 & 2.07 & 3.91 \\
\hline \hline
\end{tabular}
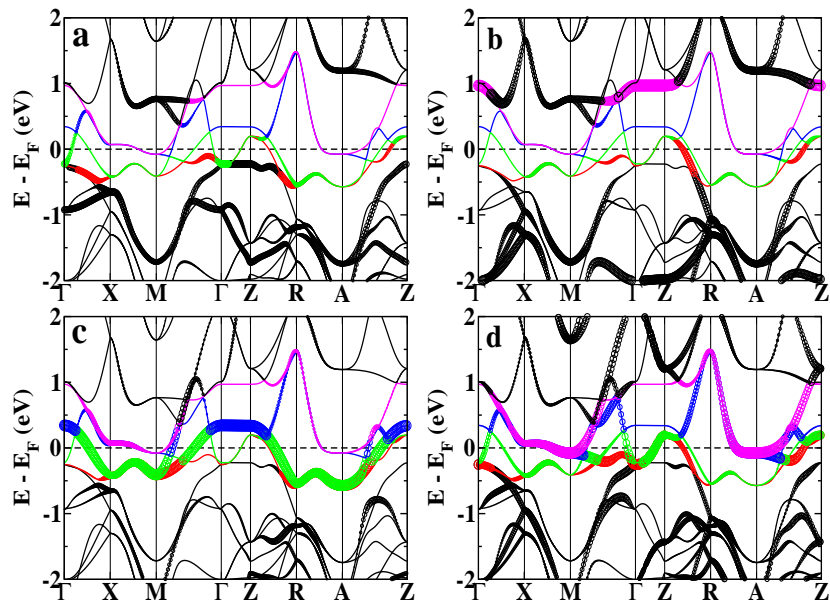

FIG. 2: (Color online) Projected band structure of CuFeAs in the NM configure, where the symbol size corresponds to the projected weight of Bloch states onto the Fe (a) $d_{r^{2}-3 z^{2}}$, (b) $\mathrm{d}_{x^{2}-y^{2}}$, (c) $\mathrm{d}_{x y}$, and (d) $\mathrm{d}_{y z / z x}$ orbits, with GGA formula. The four bands crossing the $\mathrm{E}_{F}$ are separately noted by the different colors.
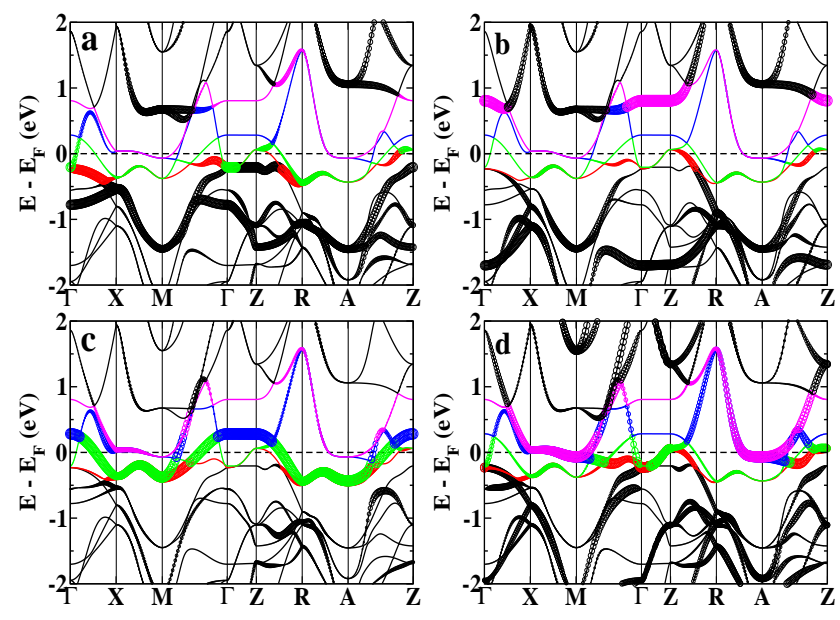

FIG. 3: (Color online) Projected band structure of CuFeSb in the NM configure, where the symbol size corresponds to the projected weight of Bloch states onto the Fe (a) $\mathrm{d}_{r^{2}-3 z^{2}}$, (b) $\mathrm{d}_{x^{2}-y^{2}}$, (c) $\mathrm{d}_{x y}$, and (d) $\mathrm{d}_{y z / z x}$ orbits, with GGA formula. The four bands crossing the $\mathrm{E}_{F}$ are separately noted by the different colors.
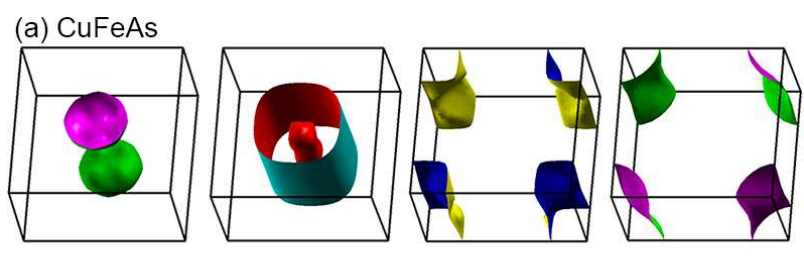

(b) CuFeSb

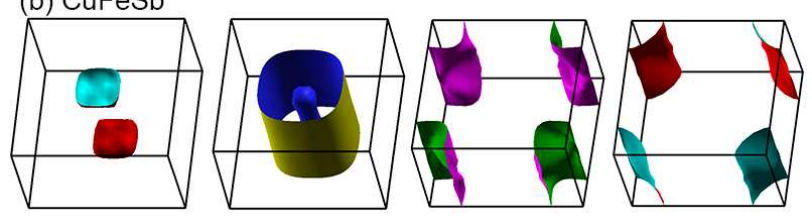

FIG. 4: (Color online) The Fermi surfaces of (a) CuFeAs and (b) CuFeSb, each band is shown separately .

of $\mathrm{d}_{x^{2}-y^{2}}$ and $\mathrm{d}_{r^{2}-3 z^{2}}$ have very little contribution [Figs. 2(a) and 2(b)]. Such orbits character is similar to the conventional iron-based materials. The projected band structure of $\mathrm{CuFeSb}$ (Fig. 3) is similar to that of CuFeAs.

The FS topology is an important issue in the ironbased superconductors ${ }^{11,13-15}$, so we present the FS of $\mathrm{CuFeAs}$ and $\mathrm{CuFeSb}$ in Figs 4(a) and 4(b), respectively. There are five FS sheets, originating from four bands across the $\mathrm{E}_{F}$ (Figs. 2 and 3 ). Their $2 \mathrm{D}$ character reflects the anisotropic electronic structure, in consistent with the anisotropic resistivity $\left(\rho_{c} / \rho_{a b} \approx 8.2\right)^{29}$. In combination with Fig. 2 (Fig. 3), the three-dimensional (3D) character plate-shaped sheet around Z-point is hole type and contributed by the mixture of three $3 \mathrm{~d}$ orbits $\left(\mathrm{d}_{x^{2}-y^{2}}, \mathrm{~d}_{z x}\right.$, and $\left.\mathrm{d}_{y z}\right)$, red and green lines in Fig. 2 (Fig. 3). Along the $\Gamma$ - $\mathrm{Z}$ line, there are two hole-like FS sheets. The small pocket shows strong 3D character, while the larger cylinder-shaped sheet shows very well $2 \mathrm{D}$ character. The small $3 \mathrm{D}$ pocket is primarily $\mathrm{d}_{y z / z x}$ characteristic, while the 2D cylinder sheet mainly has $\mathrm{d}_{x y}$ character. Except for the above mentioned three hole-like FS sheets, two electron-like FS sheets exist at the Brillouin zone corner along M-A line and display a little dispersion along $\mathrm{K}_{z}$. From Figs. 2(c) and 2(d) [Figs. 3(c) and 3(d)] we can see that these electron-like FS sheets have $\mathrm{d}_{x y}, \mathrm{~d}_{y z}$, and $\mathrm{d}_{z x}$ character, which is similar to that of $\mathrm{LiFeAs}$ and $\mathrm{MgFeGe}{ }^{37}$. 
In order to determine the true magnetic ground state of $\mathrm{CuFeAs}$ and $\mathrm{CuFeSb}$, we studied four different magnetic phases and summarized the results in Table 1. For both compounds, the FM state is the most stable state, which is consist with the experimental result of $\mathrm{CuFeSb}^{29}$. For CuFeAs, our calculated results support Qian's conclusion (FM state) ${ }^{29}$ rather than Thakur's report (AF state $)^{28}$. The calculated magnetic moments of $\mathrm{Fe}$ ions in $\mathrm{CuFeAs}$ and $\mathrm{CuFeSb}$ are comparable to the theoretical values of other iron based materials. For $\mathrm{CuFeSb}$, the experimental moment is $1.70 \mu_{B} / \mathrm{Fe}^{30}$, which is a little smaller than calculated result. However, the experiment $\mathrm{Fe}$ moment of nonstoichiometric $\mathrm{Cu}_{x} \mathrm{Fe}_{1+y} \mathrm{As}$ is only $0.33 \mu_{B} / \mathrm{Fe}^{29}$, which is rather smaller than the theoretical value. The $\mathrm{Cu}$ vacancies in $\mathrm{Cu}_{x} \mathrm{Fe}_{1+y}$ As might associate with the moment reduc$\operatorname{tion}^{29}$.

Just like $\mathrm{LaOFeAs}^{21}$, we map the total energies to the $J_{1}-J_{2}$ Heisenberg model and deduce the nearest- and next-nearest-neighbor exchange coupling interactions as

$$
J_{1}=\frac{E_{F M}-E_{A F 1}}{4 S^{2}}
$$

and

$$
J_{2}=\frac{E_{F M}+E_{A F 1}-2 E_{A F 2}}{8 S^{2}},
$$

where $E_{F M}, E_{A F 1}$, and $E_{A F 2}$ are the total energies of ferromagnetic, checkerboard, and stripe antiferromagnetic states and $S$ is the Fe spin moment. For CuFeAs $J_{1}=-32.28 \mathrm{meV}$ and $J_{2}=0.80 \mathrm{meV}$, while $J_{1}=-34.22$ $\mathrm{meV}$ and $J_{2}=-2.44 \mathrm{meV}$ for CuFeSb. It is known that when $J_{2}>\left|J_{1}\right| / 2$, the system will give way to the stripe $\mathrm{AF}$ ground state ${ }^{18-21}$. Nevertheless, for CuFeAs and $\mathrm{CuFeSb}$, the negative $J_{1}$ and relatively weak $J_{2}$ results in $J_{2} \ll J_{1} / 2$, which means that the FM state is more favorable. These exchange coupling interactions are consistent with the tendency towards ferromagnetism determined by the total energy calculation.

Fig. 5 shows the DOS and projected DOS of CuFeAs [Fig.5(a) and 5(b)] and CuFeSb [Fig.5(c) and 5(d)] in the FM state, where their DOS are similar to each other. As illustrated in Fig.5, both Cu-3d states and As-4p (or Sb-5p) states distribute deeply blow the $\mathrm{E}_{F}$. Around the $E_{F}$, the states are mainly derived from the Fe-3d states, with up spin states being almost fully occupied and down spin states partially occupied. Compared with the NM state, the $\mathrm{N}\left(\mathrm{E}_{F}\right)$ of FM state substantially reduces, while the $\mathrm{N}\left(\mathrm{E}_{F}\right)$ of the $\mathrm{AF}$ states does not decrease. According to the Stoner mechanism ${ }^{35}$, these compounds will favor the FM state, just like other itinerant magnetic systems $\mathrm{YFe}_{2} \mathrm{Ge}_{2}{ }^{39}$ and $\mathrm{CuFe}_{2} \mathrm{Ge}_{2}{ }^{40}$.

Previous studies indicate that the anion height $\left(\mathrm{Z}_{\text {anion }}\right)$ is important to determinate the electronic
TABLE II: Calculated the nearest- and next-nearest-neighbor exchange coupling interactions $J_{1}, J_{2}$, and $\left|J_{1}\right| / 2$, versus $\mathrm{Sb}$ height.

\begin{tabular}{cccc}
\hline \hline Sb height $(\AA)$ & $J_{1}(\mathrm{meV})$ & $J_{2}(\mathrm{meV})$ & $J_{1} \mid / 2(\mathrm{meV})$ \\
\hline 1.64 & 2.38 & 12.65 & 1.19 \\
1.69 & -8.33 & 8.99 & 4.17 \\
\hline 1.74 & -18.34 & 5.16 & 9.17 \\
1.79 & -27.03 & 1.27 & 13.52 \\
1.84 & -34.22 & -2.44 & 17.11 \\
1.89 & -40.43 & -5.92 & 20.21 \\
\hline \hline
\end{tabular}

structure and even the magnetic ordering in the iron based materials ${ }^{25,27,41,42}$. The large $\mathrm{Z}_{\text {anion }}$ is the remarkably character of CuFeAs and CuFeSb. To investigate the effect of $\mathrm{Z}_{\text {anion }}$ on magnetic ordering, we studied the stable energy and the Fe moment as a function of $\mathrm{Z}_{S b}$ and presented the results in Fig.6. At the experimental height $\mathrm{Z}_{S b}=1.84 \AA$, FM is the most stable state. When $\mathrm{Z}_{S b}$ is decreased to $1.74 \AA$ (experimental height $\mathrm{Z}_{A s}$ of $\mathrm{CuFeAs}$ ), the energy difference between FM and AF2 states reduces, although FM is still the most stable state. Further decreasing $\mathrm{Z}_{S b}$, the $\mathrm{FM}$ and AF2 states become degenerate at $\mathrm{Z}_{S b}=1.72 \AA$, and finally the AF2 state becomes more stable than FM state for $\mathrm{Z}_{S b}<1.72 \AA$. The special value $\mathrm{Z}_{S b}=1.72 \AA$ is a Quantum Critical Point (QCP), where the compound translate from the FM state to stripe AF state. Such transition has also been predicated by $\mathrm{Moon}^{27}$ in the FeTe compound. They found that FeTe switched its ground state from the stripe AF state to double-stripe AF state at $\mathrm{Z}_{T e}=1.71 \AA^{27}$. The Fe magnetic moment varying with $\mathrm{Z}_{S b}$ is presented in Fig.6(b), which is in agreement with the magnetic moment dependence on the $\mathrm{Z}_{\text {anion }}$ of other iron-based superconductors. The $\mathrm{Z}_{\text {anion }}$ of LaOFeAs, $\mathrm{BaFe}_{2} \mathrm{As}_{2}$, and FeTe are 1.31, 1.35, and $1.73 \AA$, respectively ${ }^{1,2,8}$. At the same time, the Fe moments are $0.36 \mu_{B}$ for $\mathrm{LaOFeAs}^{43}, 0.87 \mu_{B}$ for $\mathrm{BaFe}_{2} \mathrm{As}_{2}{ }^{44}$, and $1.70 \mu_{B}$ for $\mathrm{FeTe}^{8}$.

To study how the $\mathrm{Z}_{S b}$ influences on magnetic order, we map the total energies of different magnetic states to the $J_{1}-J_{2}$ Heisenberg model and deduce the exchange coupling interactions $J_{1}$ and $J_{2}$ as a function of $\mathrm{Z}_{S b}$. In this model, the energy differences between various magnetic orderings are assumed predominantly by the contribution from the exchange interactions between the Fe moments with spin $\hat{S}$. So the Hamilton can be effectively modeled by the following the Heisenberg model:

$$
H=J_{1} \sum_{<i j>} \overrightarrow{S_{i}} \cdot \overrightarrow{S_{j}}+J_{2} \sum_{<<i j>>} \overrightarrow{S_{i}} \cdot \overrightarrow{S_{j}}
$$

where $\langle i j\rangle$ and $<<i j>>$ denote the summation over the nearest- and next-nearest-neighbors, respectively. Though the contribution of itinerant electrons was 

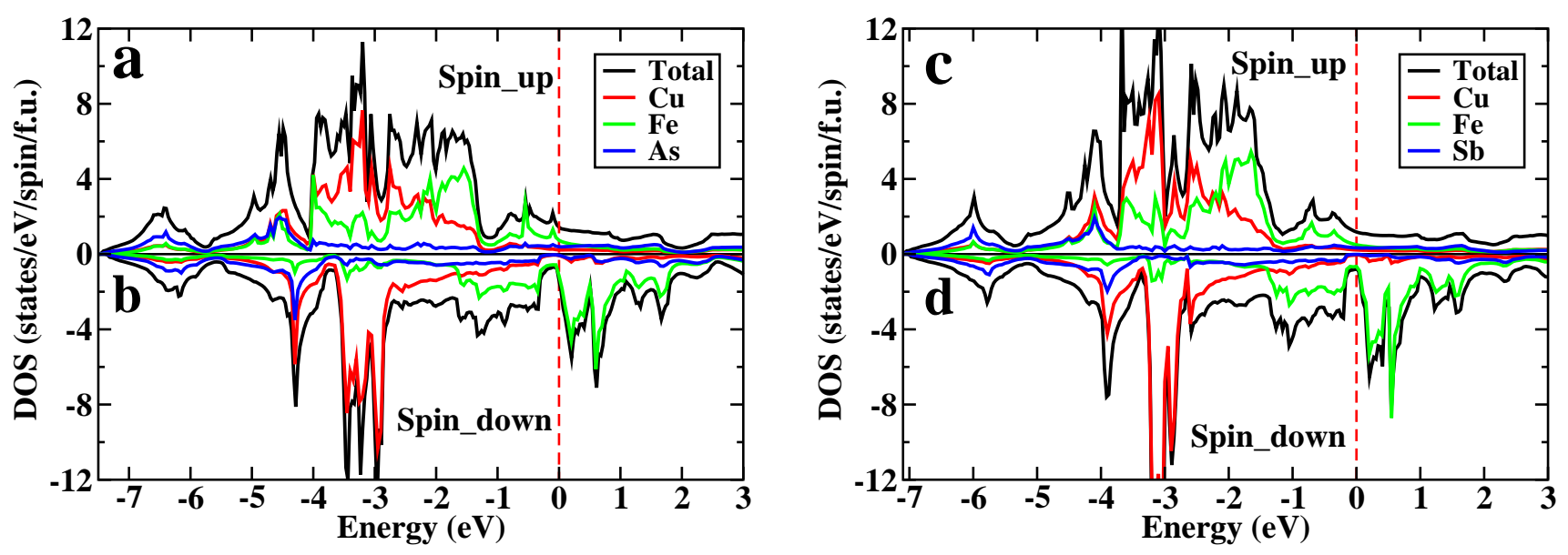

FIG. 5: (Color online) The DOS and projected DOS of (a, b) CuFeAs and (c, d) CuFeSb in the FM configure, with GGA formula.

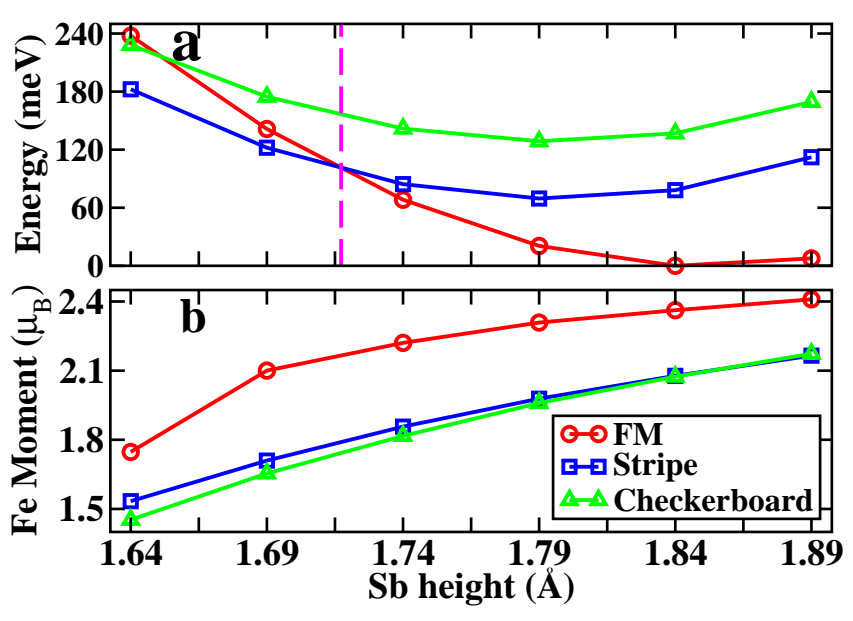

FIG. 6: (Color online) (a) The relative total energy with respect to the FM ground state $\left(\mathrm{Z}_{S b}=1.84 \AA\right)$ and $(\mathrm{b})$ the magnetic moment of Fe versus $\mathrm{Sb}$ height calculated for the FM, AF1, and AF2 phases.

missed in this model and our system was still away from the fully localized insulating region, previous study confirmed that it captured the substantial physics on the magnetic structures of iron based materials ${ }^{18-22}$. The variations of $J_{1}$ and $J_{2}$ as a function of $\mathrm{Z}_{S b}$ are summarized in Table 2. We find that the magnetic phase can be divided into two parts: 1 . in the range $1.74 \AA$ $\leq \mathrm{Z}_{S b} \leq 1.89 \AA$, wherer $J_{1}<0$ and $J_{2}<\left|J_{1}\right| / 2$, the compounds are in the FM state; 2 . for $\mathrm{Z}_{S b}<1.74 \AA$, where $J_{2}>\left|J_{1}\right| / 2$, the stripe $\mathrm{AF}$ is the ground state. A similar FM-to-AF2 transition and $J_{1}-J_{2}$ behavior were also found in $\mathrm{CuFeAs}$, but the critical value is $\mathrm{Z}_{A s}$ $=1.66 \AA$, smaller than $\mathrm{Z}_{S b}=1.72 \AA$ in $\mathrm{CuFeSb}$ and $\mathrm{Z}_{T e}=1.71 \AA$ in $\mathrm{FeTe}^{27}$. The small radius of As ion compared to $\mathrm{Sb}$ and $\mathrm{Te}$ ions might be the reason for the smaller critical value $\mathrm{Z}_{A s}$.
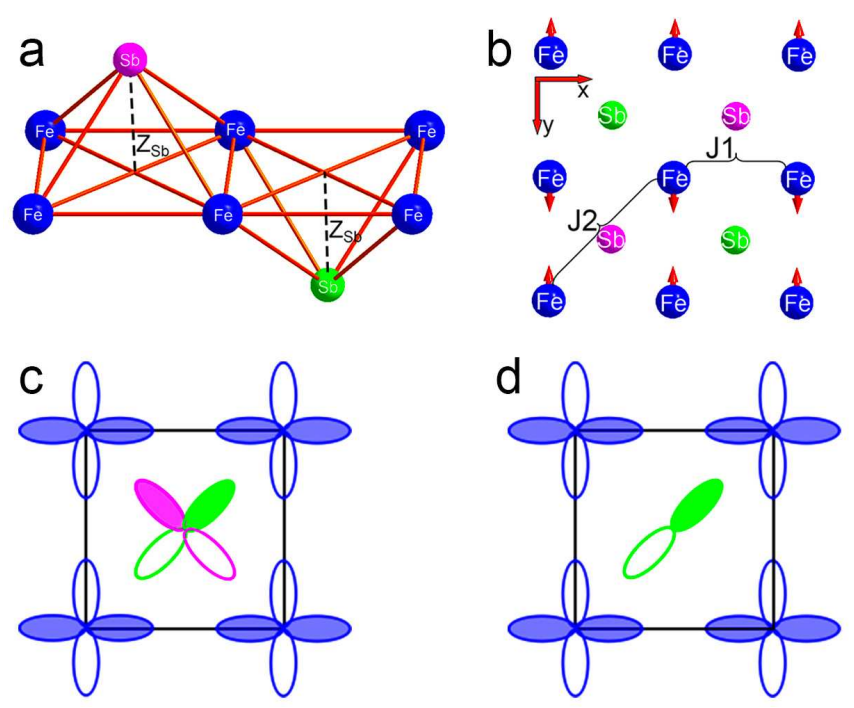

FIG. 7: (Color online) (a) Atomic structure of the FeSb layer, where $\mathrm{Z}_{S b}$ is distance from $\mathrm{Sb}$ atom to the Fe atoms plane. (b) The dominant super-exchange actions are those between both a pair of nearest-neighbor (NN) Fe spins $\left(J_{1}\right)$ and a pair of next-nearest-neighbor (NNN) Fe spins $\left(J_{2}\right)$. The process contributing to the NNN super-exchange interaction between a pair of electrons. It involves the same $4 \mathrm{p}$ orbital, leading to an $\mathrm{AF} J_{2}$. The process contributing to (c) d-d NN interaction $J_{1}(\mathrm{FM})$ and (d) d-p-d NNN interaction $J_{2}(\mathrm{AF})$.

The reason why reducing $\mathrm{Z}_{S b(A s)}$ will decrease the $\mathrm{NN}$ interaction $J_{1}$ but increase the NNN interaction $J_{2}$ can be understood by the schematic diagram of Fig.7. When the $\mathrm{Z}_{S b(A s)}$ is shortened, the $\mathrm{d}-\mathrm{p}-\mathrm{d} \mathrm{NNN}$ interaction $J_{2}(\mathrm{AF})$ will increase because that the $\mathrm{P}_{x / y}$-orbits of $\mathrm{Sb}(\mathrm{As})$ atom come nearly to the Fe-atoms plane and then enhance the d-p-d exchange integral. But the 
d-d NN interaction $J_{1}(\mathrm{FM})$ will be reduced when the $\mathrm{Sb}(\mathrm{As})$ atoms come nearly to the Fe-atoms plane. At some special $\mathrm{Z}_{S b(A s)}$ value, i.e. the Quantum Critical Point (QCP), the compounds will translate from the FM state to stripe AF state.

\section{SUMMARY}

In summary, by the first-principles calculations, we studied the electronic structures and magnetism of $\mathrm{CuFeAs}$ and $\mathrm{CuFeSb}$. In the nonmagnetic state, their DOS, orbits character, band structure, and Fermi surface show similarities to those of other iron based superconductors. The total energy calculations indicate that the ground state of these materials is the ferromagnetic state. Such ferromagnetic ground state is owning to the large anion height $\mathrm{Z}_{\text {anion }}$, which reduces the d-P-d NNN interaction $J_{2}(\mathrm{AF})$ and increases d-d $\mathrm{NN}$ interaction $J_{1}(\mathrm{FM})$. The anion height $\mathrm{Z}_{\text {anion }}$ is the key tuning parameter for these two competing magnetic interactions (AF and $\mathrm{FM}$ ), and it can switch the ground state from the AF state to FM state.

\section{ACKNOWLEDGMENTS}

The authors acknowledge the support from NSF of China (No.11274095, No.10947001) and the high performance computing center of Henan Normal University.
1 Y. Kamihara, T. Watanabe, M. Hirano, and H. Hosono, J. Am. Chem. Soc. 130 (2008) 3296.

2 M. Rotter, M. Tegel, and D. Johrendt, Phys. Rev. Lett. 101 (2008) 107006.

3 X.C. Wang, Q.Q. Liu, Y.X. Lv, W.B. Gao, L.X. Yang, R.C. Yu, F.Y. Li, and C.Q. Jin, Solid State Commun. 148 (2008) 538.

4 G.F. Chen, W.Z. Hu, J.L. Luo, and N.L. Wang, Phys. Rev. Lett. 102 (2009) 227004.

5 X. Zhu, F. Han, G. Mu, P. Cheng, B. Shen, B. Zeng, and H.-H. Wen, Phys. Rev. B 79 (2009) 220512(R); G.T. Wang, M.P. Zhang, L.H. Zheng, and Z.X. Yang, Phys. Rev. B 80 (2009) 184501.

6 Z. Yang, Z. Liu, A. Fang, F. Huang, and X. Xie, J. Alloys Compd. 509 (2011) 2846 - 2849; J.B. Anooja, P.M. Aswathy, P.M. Sarun, and U. Syamaprasad, J. Alloys Compd. 514 (2012) 1 - 5.

7 F.C. Hsu, J.Y. Luo, K.W. Yeh, T.K. Chen, T.W. Huang, P.M. Wu, Y.C. Lee, Y.L. Huang, Y.Y. Chu, D.C. Yan, and M.K. Wu, Proc. Natl. Acad. Sci. USA 105 (2008) 14262.

8 S.L. Li, C.de.la. Cruz, Q. Huang, Y. Chen, J.W. Lynn, J.P. $\mathrm{Hu}$, Y.L. Huang, F.C. Hsu, K.W. Yeh, M.K. Wu, and P.C. Dai, Phys. Rev. B 79 (2009) 054503.

9 J. Guo, S. Jin, G. Wang, S. Wang, K. Zhu, T. Zhou, M. He, and X. Chen, Phys. Rev. B 82 (2010) 180520(R).

10 X.F. Lu, N.Z. Wang, H. Wu, Y.P. Wu, D. Zhao, X.Z. Zeng, X.G. Luo, T. Wu, W. Bao, G.H. Zhang, F.Q. Huang, Q.Z. Huang, and X.H. Chen, Nat. Mater. 15 (2015) 325.

11 I.I. Mazin, Nature. 464 (2010) 183.

12 J.P. Hu and H. Ding, Sci. Rep. 2 (2012) 381.

13 J. Dong, H.J. Zhang, G. Xu, Z. Li, G. Li, W.Z. Hu, G.F. Chen, X. Dai, J.L. Luo, Z. Fang, and N.L. Wang, Europhys. Lett. 83 (2008) 27006.

14 F.J. Ma and Z.Y. Lu, Phys. Rev. B 78 (2008) 033111.

15 I.I. Mazin, D.J. Singh, M.D. Johannes, and M.H. Du, Phys. Rev. Lett. 101 (2008) 057003.

16 W. Bao, Y. Qiu, Q. Huang, M.A. Green, P. Zajdel, M.R. Fitzsimmons, M. Zhernenkov, S. Chang, M. Fang, B. Qian, E. K. Vehstedt, J. Yang, H.M. Pham , L. Spinu, and Z.Q. Mao, Phys. Rev. Lett. 102 (2009) 247001.

17 A. Subedi, L.J. Zhang, D.J. Singh, and M.H. Du, Phys. Rev. B 78 (2008) 134514.
18 C. Fang, H. Yao, W.F. Tsai, J.P. Hu, and S.A. Kivelson, Phys. Rev. B 77 (2008) 224509.

19 T. Yildirim, Phys. Rev. Lett. 101 (2008) 057010.

20 Q.M. Si and E. Abrahams, Phys. Rev. Lett. 101 (2008) 076401 .

21 F.J. Ma, Z.Y. Lu, and T. Xiang, Phys. Rev. B 78 (2008) 224517.

22 G.T. Wang, M.P. Zhang, L.H. Zheng, and Z.X. Yang, Phys. Lett. A 376 (2012) 1072 - 1077.

23 A.L. Wysocki, K.D. Belashchenko, and V.P. Antropov, Nature Phys. 7 (2011) 485.

24 M.D. Johannes and I.I. Mazin, Phys. Rev. B 79 (2009) 220510(R).

25 W.G. Yin, C.C. Lee, and W. Ku, Phys. Rev. Lett. 105 (2010) 107004.

26 G. Xu, W. Ming, Y. Yao, X. Dai, S.C. Zhang, and Z. Fang, Europhys. Lett. 82 (2008) 67002.

27 C.Y. Moon and H.J. Choi, Phys. Rev. Lett. 104 (2010) 057003 .

28 G.S. Thakur, Z. Haque, L.C. Gupta, and A.K. Ganguli, J. Phys. Soc. Jpn. 83 (2014) 054706.

29 B. Qian, J. Hu, J. Liu, Z. Han, P. Zhang, L. Guo, X. Jiang, T. Zou, M. Zhu, C.de.la. Cruz, X. Ke, and Z.Q. Mao, Phys. Rev. B 91 (2015) 014504.

30 B. Qian, J. Lee, J. Hu, G.C. Wang, P. Kumar, M.H. Fang, T.J. Liu, D. Fobes, H. Pham, L. Spinu, X.S. Wu, M. Green, S.H. Lee, and Z.Q. Mao, Phys. Rev. B 85 (2012) 144427.

31 Z. Fang and K. Terakura, J. Phys.: Condens. Matter. 14 (2002) 3001.

32 J.P. Perdew and Y. Wang, Phys. Rev. B 45 (1992) 13244.

33 J.P. Perdew, K. Burke, and M. Ernzerhof, Phys. Rev. Lett. 77 (1996) 3865.

34 D.J. Singh, Phys. Rev. B 79 (2009) 153102; B. Cheng, B.F. Hu, R.Y. Chen, G. Xu, P. Zheng, J.L. Luo, and N.L. Wang, Phys. Rev. B 86 (2012) 134503; J.A. McLeod, E.Z. Kurmaev, I. Perez, V.K. Anand, P.K. Perera, D.C. Johnston, and A. Moewes, Phys. Rev. B 88 (2013) 014508.

35 O.K. Andersen, J. Madsen, U.K. Poulsen, O. Jepsen, and J. Kollár, Physica B 86 (1977) 249; G.L. Krasko, Phys. Rev. B 36 (1987) 8565.

36 D.J. Singh and M.-H. Du, Phys. Rev. Lett. 100 (2008) 237003; G.T. Wang, L.H. Zheng, M.P. Zhang, and Z.X. 
Yang, Phys. Rev. B 81 (2010) 014521.

37 X.F. Liu, S. Matsuishi, S. Fujitsu, and H. Hosono, Phys. Rev. B 85 (2012) 104403; Rhee H. B. and Pickett W. E., J. Phys. Soc. Jpn. 82 (2013) 034714.

38 H.O. Jeschke, I.I. Mazin, and R. Valentí, Phys. Rev. B 87 (2013) 241105(R).

39 D.J. Singh, Phys. Rev. B 89 (2014) 024505; A. Subedi, Phys. Rev. B 89 (2014) 024504.

${ }^{40}$ K.V. Shanavas and D.J. Singh, PloS one 10 (2015) e0121186.

41 M.J. Winiarski, M. Samsel-Czekala, and A. Ciechan, J. Alloys Compd. 566 (2013) 187 - 190.
42 M. Monni, F. Bernardini, G. Profeta, and S. Massidda Phys. Rev. B 87 (2013) 094516; A. Ciechan, M. J. Winiarski, and M. Samsel-Czekala, J. Phys.: Condens. Matter. 26 (2014) 025702.

43 C.de.la.z. Cru, Q. Huang, J.W. Lynn, J. Li, W. Ratcliff II, J.L. Zarestky, H.A. Mook, G.F. Chen, J.L. Luo, N.L. Wang, and P.C. Dai, Nature (London) 453 (2008) 899.

44 Q. Huang, Y. Qiu, W. Bao, M.A. Green, J.W. Lynn, Y.C. Gasparovic, T. Wu, G. Wu, and X.H. Chen, Phys. Rev. Lett. 101 (2008) 257003. 